\title{
High expression of CDCA7 predicts tumor progression and poor prognosis in human colorectal cancer
}

\author{
SIMAN LI $^{1 *}$, JIEAN HUANG ${ }^{2 *}$, MENGBIN QIN $^{2}$, JINXIU ZHANG $^{2}$ and CUN LIAO ${ }^{3}$ \\ ${ }^{1}$ Department of Gastroenterology, The First Affiliated Hospital of Guangxi Medical University, Nanning, \\ Guangxi 530021; ${ }^{2}$ Department of Gastroenterology, The Second Affiliated Hospital of Guangxi Medical University, \\ Nanning, Guangxi 530007; ${ }^{3}$ Department of Colorectal-Anal Surgery, The First Affiliated Hospital of \\ Guangxi Medical University, Nanning, Guangxi 530021, P.R. China
}

Received April 13, 2019; Accepted February 7, 2020

DOI: $10.3892 / \mathrm{mmr} .2020 .11089$

\begin{abstract}
Colorectal cancer (CRC) is one of the most fatal types of cancer worldwide. This study aimed to determine the predictive and prognostic values of cell division cycle associated protein 7 (CDCA7) in CRC. Firstly, the relationship between CDCA7 and CRC was assessed through bioinformatics analysis. Subsequently, CDCA7 expression levels were detected in various CRC cell lines, as well as 15 fresh human CRC tissues and their paired adjacent normal colorectal tissues using reverse transcription-quantitative PCR and western blotting. Additionally, immunohistochemical staining was used to determine the levels of CDCA7 in 104 CRC tissues and their paired adjacent normal colorectal tissues. The present study revealed that CDCA7 expression was upregulated in CRC tissues and cell lines. The positive expression rates of CDCA7 in normal and CRC tissues were 26.92 and $75.96 \%$, respectively. The intensities of $\mathrm{CDCA} 7$ immunostaining were significantly associated with CRC invasion depth, lymph node metastasis, tumor-node-metastasis stage and distant metastasis. However, no significant differences in sex, age, tumor size and CRC differentiation were found between high and low CDCA7 expression groups. Furthermore, patients with low CDCA7 expression exhibited a greater overall survival rate of CRC compared to those with high CDCA7 expression. The findings of this study indicated that CDCA7 may serve a significant role in CRC prognosis and progression, and may be considered a novel biomarker for the prediction of patient survival after colectomy.
\end{abstract}

Correspondence to: Dr Cun Liao, Department of Colorectal-anal Surgery, The First Affiliated Hospital of Guangxi Medical University, 6 Shuangyong Road, Nanning, Guangxi 530021, P.R. China

E-mail: 1scnn2000@hotmail.com

${ }^{*}$ Contributed equally

Key words: cell division cycle associated protein 7, colorectal cancer, progression, prognosis, bioinformatics analysis

\section{Introduction}

Colorectal cancer (CRC) is recognized as one of the most common and deadly cancers worldwide (1). It occupies an increasingly important position in the spectrum of cancer, and represents the leading cause of cancer-related mortality worldwide $(2,3)$. Due to the lack of specific clinical symptoms and early screening tests, a substantial number of patients with CRC are first diagnosed at an advanced stage with metastasis (4). Furthermore, even if a tumor is resected, CRC recurrence and metastasis may occur after surgery; therefore, the prognosis of CRC remains poor (5). Thus, it is of great importance to understand the molecular mechanisms underlying CRC development and to identify novel tumor-associated biomarkers.

Bioinformatics analysis provides a deeper and more comprehensive understanding of targeted genes, and allows researchers to identify functional genes for further analysis. The Cancer Genome Atlas (TCGA) generates comprehensive, multi-dimensional maps of the key genomic changes in cancer, and provides researchers with a variety of genetic information on different types of cancer (6). Kyoto Encyclopedia of Genes and Genomes (KEGG) (7) and Gene Ontology (GO) (8) analyses have enabled the identification of metabolic signaling pathways and major biological functions of differentially expressed genes. Therefore, TCGA, data collection, and GO and KEGG pathway enrichment analyses were performed in this study.

Cell division cycle associated protein 7 (CDCA7) is located on chromosome $2 \mathrm{q} 31$ and encodes a nuclear protein containing 371 amino acids (9). CDCA7 is a cell division cycle-associated protein that was first discovered in Myc-transfected fibroblasts, which is commonly overexpressed in various types of human cancer (10). CDCA7 is periodically expressed during the human cell cycle, with the highest expression level found in the G1 to S phase (11). Deregulation of cell cycle proteins often leads to the increased risk of tumor occurrence $(12,13)$. Previous reports have demonstrated that CDCA7 is a c-Myc-responsive gene that participates in cancer transformation and tumorigenesis (9). Thus far, several studies have reported that CDCA7 is overexpressed in different types of cancer, including lymphoma, ovarian cancer, retinoblastoma, breast cancer, acute myeloid leukemia and esophageal 
cancer (10,14-18). These findings provide evidence that there may be an association between CDCA7 and tumor development. However, to the best of our knowledge, the relationship between CDCA7 and the development of colorectal disease has not yet been reported on. Therefore, the clinical relevance and underlying mechanisms of CDCA7 in the occurrence and development of CRC remain largely unclear. The present study aimed to investigate the expression levels and clinical role of CDCA7 in CRC.

\section{Materials and methods}

\section{Bioinformatics analysis}

Collection of CDCA7 expression data from TCGA. microRNA (miR/miRNA) expression matrix was retrieved from TCGA (https://portal.gdc.cancer.gov). RNA-seq data from 647 CRC tissues (cancer group) and 51 normal adjacent tissues (normal group) were downloaded from TCGA database (dataset nos. TCGA-COAD and TCGA-READ). Differentially expressed levels of CDCA7 between the cancer group and normal group were identified using the Ballgown (https://github.com/alyssafrazee/ballgown) package with the following criteria: $\mathrm{P}<0.05$ and fold-change $>2$.

Gene functions and pathways. KEGG (http://www.genome. jp/kegg/pathway.html) and GO (http://geneontology.org/) were used for the enrichment analysis of dysregulated genes, in order to determine the changes in functions and pathways associated with CRC. The conditional criterion was $\mathrm{P}<0.05$.

Research hotspots. According to the gene annotation in the database, genetic studies were evaluated based on five different perspectives: i) Relevant literature of CDCA7 (total number of articles reported); ii) pathways (number of pathways that CDCA7 was involved in according to the KEGG database); iii) functions (number of functions that involved the CDCA7 gene according to the GO database); iv) validated targeted miRNAs (number of miRNAs associated with CDCA7 that have been published in literature); and v) diseases (number of diseases associated with CDCA7 that have been published in the literature), in order to identify research hotspots of CDCA7 through the KEGG and GO databases, and published literature. A radar chart was produced using Microsoft Office 2013 software to demonstrate the research hotspots of CDCA7 (Fig. 1).

Disease association. The frequency of the gene reported in different diseases was mined from the PubMed database (https://www.ncbi.nlm.nih.gov/pubmed/) according to the MeSH disease classification (https://www.ncbi.nlm.nih.gov/mesh). The top 20 diseases associated with the target gene were sought out.

Regulatory relationship. The regulatory relationship among genes was examined based on four different perspectives: Gene-related transcription factors, miRNAs, long non-coding (lnc)RNAs, as well as upstream and downstream gene sequences, via published literature and the databases of PubMed, MeSH and KEGG.

Transcription factor prediction. Transcription factors were predicted according to the positional binding patterns. The integrated score based on the two predicted scores in the TRANSFAC ${ }^{\circledR}$ database (http://genexplain. com/transfac/\#section3) were assigned as the final predicted score. Binding regions of the predicted transcription factors were annotated based on the DNA methylation and single nucleotide polymorphism (SNP) information in COSMIC (19) and dbSNP (https://www.ncbi.nlm.nih.gov/snp/) databases, respectively. According to the predicted scores of the TRANSFAC ${ }^{\circledR}$ database and whether the corresponding comment information existed in the COSMIC database and the dbSNP database, the recommendation degree was integrated. The recommendation degree was used to represent the research value of the predicted transcription factor; the higher the recommendation degree, the better. Results with a high recommendation degree were selected for use in the present study.

Gene expression in tumors. According to TCGA barcodes, all samples were classified into normal and tumor tissues. Subsequently, the average value and standard deviation of all samples were determined based on the expression values in the normalized-results file of each sample provided by RNA-SEQv2 data. Finally, the target gene expression in human tumors was interpreted.

Tissue samples. CRC tissues and the paired adjacent non-tumor tissues $(n=104)$ in the form of pathological slices were collected from patients (sex, 61 males and 43 females; age, 26-82 years; average age, $56.42 \pm 12.87$ years) who underwent surgical operation at the Department of Colorectal-anal Surgery, The First Affiliated Hospital of Guangxi Medical University (Guangxi, China) between October 2012 and November 2013. In addition, CRC tissues were collected from 15 patients (sex, 9 males and 6 females; age, 47-75 years; average age, $60.33 \pm 8.83$ years) with pathological confirmation of the diagnosis in November 2017. All tissue specimens were collected within $30 \mathrm{~min}$ after surgical resection. The 15 pairs of CRC tissues and normal adjacent tissues were immediately snap-frozen in liquid nitrogen, and then stored at $-80^{\circ} \mathrm{C}$ until protein and RNA extraction. The survival status of patients with CRC was verified by telephone interview. None of the patients received pre-operative chemoradiotherapy or radiotherapy. Clinicopathological characteristics of the patients were assessed by reviewing patients' medical records and pathology reports.

The ethical approval for this study was obtained from the Protection of Human Ethics Committee of The First Affiliated Hospital of Guangxi Medical University. All specimens were made anonymous and handled in accordance with the legal and ethical standards for protecting human rights.

Cell lines and cell culture. Human normal colonic epithelial cell line NCM460 and human CRC cell line SW620 were obtained from Wuhan Boster Biological Technology, Ltd. Other human CRC cell lines, HT29, HCT116 and RKO, were purchased from Shanghai R\&S Biotechnology Co., Ltd. These cell lines were cultured in DMEM (Gibco; Thermo Fisher Scientific, Inc.) supplemented with FBS (100 ml/l; Shanghai ExCell Biology, Inc.) and maintained in $5 \% \mathrm{CO}_{2}$ at $37^{\circ} \mathrm{C}$. Mycoplasma testing was then performed 


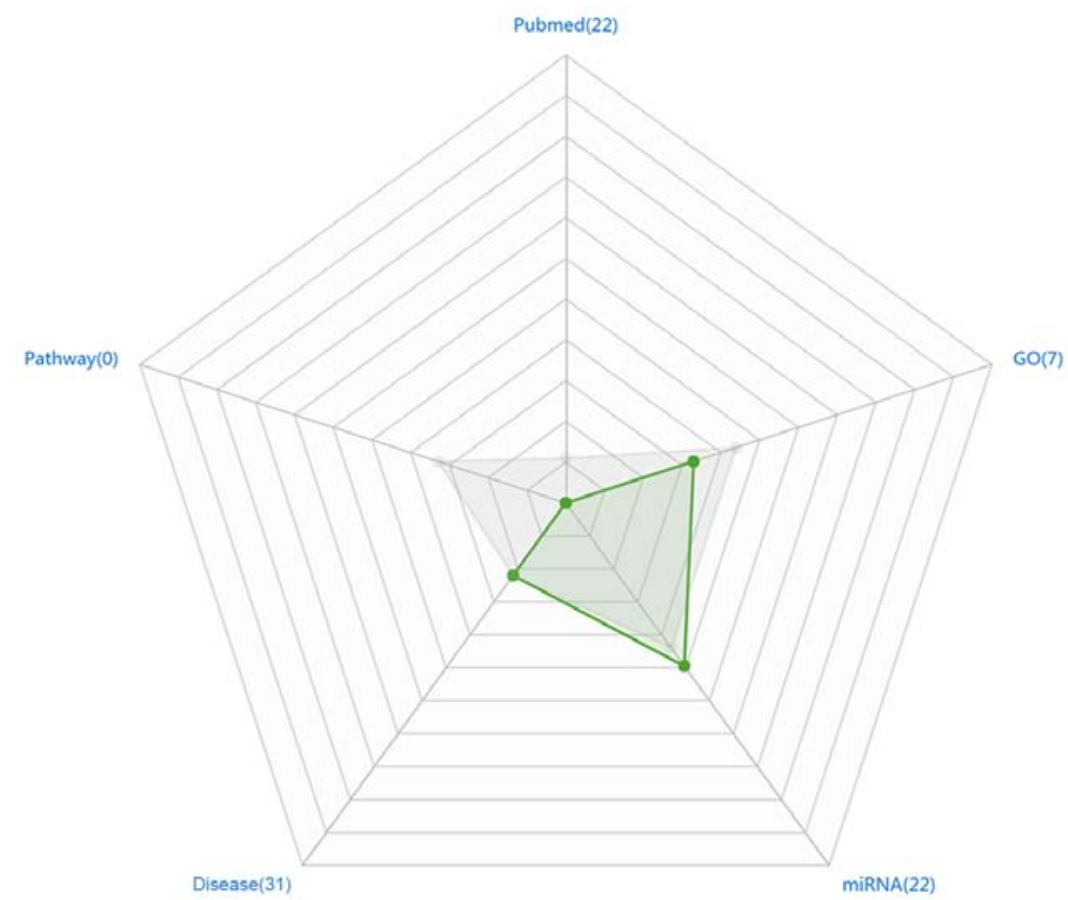

Figure 1. Research hotspots of cell division cycle associated protein 7. GO, Gene Ontology; miRNA, microRNA. The gray area represents the average number of research fever of all genes, while the green area represents the number of research fever of CDCA7. The dots represent the reported amount of CDCA7 in the field.

on all the cultured cell lines. The four human CRC cell lines were referenced to the NCM460 cell line, which acted as the control group.

Immunohistochemistry. Immunohistochemistry was performed using SPlink Detection kits (cat. no. SP-9000; OriGene Technologies, Inc.), according to the manufacturer's protocol. Tissue specimens were fixed in $10 \%$ formalin for $48 \mathrm{~h}$ at room temperature. After dehydration and paraffin embedding, all the tissue specimens were cut into $4-\mu \mathrm{m}$ sections. Specifically, at room temperature, colon tissue sections were routinely dewaxed with xylene and dehydrated in a descending alcohol series, prior to being incubated in $0.01 \mathrm{M}$ citrate buffer at $100^{\circ} \mathrm{C}$ for $6 \mathrm{~min}$ for antigen retrieval. Following being cooled down, the sections were washed three times in PBS for $5 \mathrm{~min}$ each and the sections were then incubated with $3 \% \mathrm{H}_{2} \mathrm{O}_{2}$ at room temperature for $12 \mathrm{~min}$. Subsequently, after being washed thrice, sections were incubated with goat serum (provided by the kit) at room temperature for $12 \mathrm{~min}$. Following being blocked, the tissue sections were incubated with CDCA7 polyclonal primary antibody (1:100; cat. no. PA5-52165; Invitrogen; Thermo Fisher Scientific, Inc.) at $4^{\circ} \mathrm{C}$ overnight. The following day, the incubated tissue sections were washed three times with PBS after being left at room temperature for $\geq 30 \mathrm{~min}$. The primary antibody was replaced with PBS as a negative control. Subsequently, the tissue sections were incubated with a biotin-conjugated secondary antibody (provided by the kit) at room temperature for $12 \mathrm{~min}$. Subsequently, after being washed with PBS three times, the tissue sections were incubated with horseradish peroxidase-conjugated streptomycin avidin (provided by the kit) at room temperature for $12 \mathrm{~min}$. The sections were then repeatedly immersed 3 times in PBS for $5 \mathrm{~min}$ each. After drying the sections at room temperature, the sections were stained with DAB reagent for $6 \mathrm{~min}$, counterstained with hematoxylin for $30 \mathrm{sec}$ and sealed with neutral gum. Significant yellow or yellow-brown granules appeared in the cell membrane, cytoplasm or nucleus, which was regarded as positive streptavidin peroxidase (SP) staining. Semi-quantitative analysis was conducted by integrating the staining intensity and the percentage of positive cells. The stained tissue sections were observed under an Olympus optical microscope (magnification, $\mathrm{x} 400$ ). The values of staining index (0-12) were calculated by multiplying the two scores obtained (intensity and percentage). The staining intensities of the positive cells were categorized as follows: 0 point (negative); 1 point (weak); 2 points (moderate); and 3 points (strong). A total of five different fields were randomly observed for each slice, and the percentages of positively-stained cells were categorized as follows: 0 points $(<5 \%)$; 1 point $(5-25 \%)$; 2 points $(26-50 \%) ; 3$ points $(51-75 \%)$; and 4 points $(>75 \%)$. Final staining scores of 0-7 indicated low CDCA7 expression, whereas staining scores of 8-12 represented high CDCA7 expression. Each slide was independently examined by two experienced pathologists.

RNA extraction, $c D N A$ synthesis and reverse transcription-quantitative $P C R(R T-q P C R)$. Total RNA was extracted from 30 samples of fresh $C R C$ tissues and adjacent normal colorectal tissues, as well as CRC cell lines using Eastep ${ }^{\circledR}$ Super Total RNA Extraction kit (Promega Corporation), according to the manufacturer's protocol. Subsequently, cDNA was synthesized by the GoScript ${ }^{\mathrm{TM}}$ Reverse Transcription Mix, Random Primers kit (Promega Corporation). The following RT temperature protocol was used: $5 \mathrm{~min}$ at $25^{\circ} \mathrm{C}, 60 \mathrm{~min}$ at $42^{\circ} \mathrm{C}$ and $15 \mathrm{~min}$ at $70^{\circ} \mathrm{C}$, prior to being maintained at $4^{\circ} \mathrm{C}$. RT-qPCR was conducted 
Table I. Primer sequences used for reverse transcription-quantitative PCR.

\begin{tabular}{ll}
\hline Gene & \multicolumn{1}{c}{ Primer sequences $\left(5^{\prime} \rightarrow 3^{\prime}\right)$} \\
\hline GAPDH & F: GCACCGTCAAGGCTGAGAAC \\
& R: TGGTGAAGACGCCAGTGGA \\
CDCA7 & F: CCAGGCTCCGACTCACAATCAAG \\
& R:GTACTTATCCTCTTCCTCCTCCTCCTC
\end{tabular}

CDCA7, cell division cycle associated protein 7; F, forward; R, reverse.

Table II. Gene function and pathway analysis of CDCA7.

\begin{tabular}{ll}
\hline Gene ID & \multicolumn{1}{c}{ GO function } \\
\hline GO:0006351 & Regulation, DNA template \\
GO:0006355 & Transcriptional regulation, DNA template \\
GO:0006915 & Apoptosis \\
GO:0042127 & Regulates cell proliferation \\
\hline
\end{tabular}

CDCA7, cell division cycle associated protein 7; GO, Gene Ontology.

Table III. miRNAs and lncRNAs targeted by cell division cycle associated protein 7 .

\begin{tabular}{ll}
\hline miRNA & \multicolumn{1}{c}{ lncRNA } \\
\hline hsa-let-7b-5p & HCP5 \\
hsa-miR-124-3p & TP53TG1 \\
hsa-miR-124-3p & KCNQ1OT1 \\
hsa-miR-1254 & DKFZP434K028 \\
hsa-miR-1254 & DKFZP434K028 \\
hsa-miR-1271-3p & LINC00341 \\
hsa-miR-1271-3p & BIN3-IT1 \\
hsa-miR-193b-3p & DLGAP1-AS2 \\
hsa-miR-24-3p & LOC90768 \\
hsa-miR-299-5p & BAALC-AS2 \\
\hline
\end{tabular}

lncRNA, long non-coding RNA; miRNA/miR, microRNA.

with Applied Biosystems ${ }^{\mathrm{TM}} 7500$ Real-Time PCR system (Applied Biosystems; Thermo Fisher Scientific, Inc.) using a GoTaq ${ }^{\circledR}$ qPCR Master Mix kit (Promega Corporation). qPCR cycling conditions were as follows: $10 \mathrm{~min}$ at $95^{\circ} \mathrm{C}$, followed by 40 cycles of $15 \mathrm{sec}$ at $95^{\circ} \mathrm{C}$ and $60 \mathrm{sec}$ at $60^{\circ} \mathrm{C}$, and a dissociation cycle consisting of $15 \mathrm{sec}$ at $95^{\circ} \mathrm{C}, 15 \mathrm{sec}$ at $60^{\circ} \mathrm{C}$ and $15 \mathrm{sec}$ at $95^{\circ} \mathrm{C}$ (ramping up at $0.2^{\circ} \mathrm{C} / \mathrm{sec}$ ). The expression levels of CDCA7 were measured by $2^{-\Delta \Delta \mathrm{Cq}}$ method (20) using GAPDH as a standard reference, and the relative expression value was set to 1 under unstimulated conditions. The primer sequences (Takara Bio, Inc.) of GAPDH and CDCA7 are presented in Table I.
Western blot analysis. Total protein was isolated from CRC tissues, normal colorectal tissues and CRC cell lines using RIPA lysis buffer containing $1 \%$ phosphatase inhibitor and $1 \%$ protease inhibitor. After incubation at $0^{\circ} \mathrm{C}$ for $30 \mathrm{~min}$, the lysed cells were washed twice with ice-cold PBS, and then centrifuged at $12,000 \mathrm{xg}$ and $4^{\circ} \mathrm{C}$ for $15 \mathrm{~min}$ to eliminate potential cell debris. Total protein concentration was determined by a bicinchoninic acid assay (Beyotime Institute of Biotechnology). Subsequently, the quantified proteins $(50 \mu \mathrm{g})$ were denatured and subjected to SDS-PAGE on $12 \%$ gels, and transferred onto $0.22 \mu \mathrm{m}$ PVDF membranes. The membranes were then blocked with non-fat milk $(5 \%)$ for $1 \mathrm{~h}$ at $37^{\circ} \mathrm{C}$. After washing with Tris-buffered saline containing 5\% Tween-20 (TBST) three times, the membranes were incubated with rabbit polyclonal CDCA7 antibody (1:250; cat. no. PA5-52165; Invitrogen; Thermo Fisher Scientific, Inc.) and mouse monoclonal GAPDH antibody (1:2,000; cat. no. 60004-1-lg; ProteinTech Group, Inc.) antibodies. After incubation at $4^{\circ} \mathrm{C}$ overnight, the membranes were incubated with IRDye ${ }^{\circledR}$ 680RD goat anti-rabbit immunoglobulin G (IgG; 1:10,000; cat. no. 925-68071; LI-COR Biosciences) and goat anti-mouse IgG (1:5,000; cat. no. BA1038; Wuhan Boster Biological Technology, Ltd.) secondary antibodies, respectively, at room temperature for $1 \mathrm{~h}$. After washing with TBST three times, the stained membranes were visualized using an Odyssey ${ }^{\circledR}$ CLx imaging system (LI-COR Biosciences). The gray value of each protein was determined by ImageJ version 1.4.3 software (National Institutes of Health). GAPDH expression was used as the internal reference. The relative expression level of CDCA7 was calculated by the ratio of CDCA7 gray value to GAPDH gray value.

Statistical analysis. All analyses were carried out using SPSS Statistics version 20.0 (IBM Corp.). Each experiment was repeated three times. Student's paired t-test was performed to compare the expression levels of CDCA7 between CRC tissues and adjacent normal colorectal tissues. Expression levels of CDCA7 in CRC cell lines were compared using a one-way ANOVA and Bonferroni's post hoc test. The strength of association between CDCA7 expression and CRC clinicopathological features was determined using a $\chi^{2}$ test or Fisher's exact test. Overall survival data were analyzed by the Kaplan-Meier method and log-rank test. Data are presented as the means $\pm \mathrm{SD}$. $\mathrm{P}<0.05$ was regarded as statistically significant.

\section{Results}

Bioinformatics prediction of CDCA7 $m R N A$ expression in $C R C$. Based on the data obtained from TCGA database, under the criteria of $\mathrm{P}<0.05$ and $\log _{2}$ fold changel $>2$, the mRNA expression of CDCA7 was upregulated in CRC tissues compared to normal tissues $(\mathrm{P}<0.05)$, and the fold change (T/N) was 3.06.

GO and KEGG pathway enrichment analysis. The biological functions of CDCA7 in CRC were determined by GO and KEGG pathway enrichment analyses. As shown in Table II, according to GO term analysis, it was noted that the most marked functions of CDCA7 in CRC were apoptosis and 


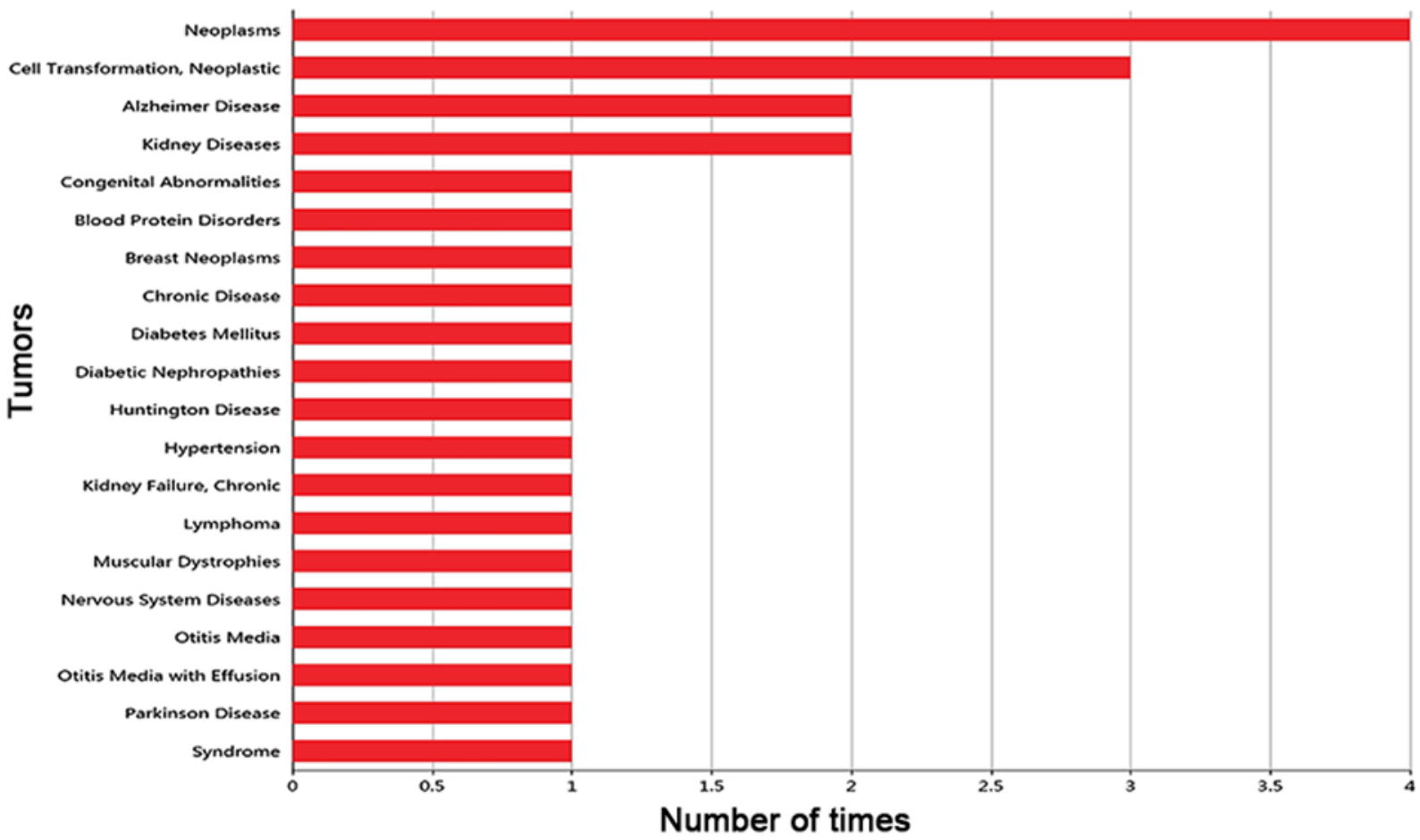

Figure 2. Top 20 most commonly reported diseases associated with cell division cycle associated protein 7 .

transcription. However, no significant finding was obtained for gene-involved pathways, as revealed by KEGG pathway analysis.

Research hotspots of CDCA7. As shown in Fig. 1, a total of 22 articles were reported on CDCA7, in which CDCA7 was associated with seven biological GO functions. However, according to the KEGG database, no signaling pathway involving CDCA7 was found. The total number of diseases associated with CDCA7 was 31, and 22 different targeted miRNAs had been validated. Only 2 (disease and miRNA) of the 5 indicators of CDCA7 were higher than average. Thus, the lack of findings surrounding CDCA7 indicated that little research has been performed on this gene until relatively recently.

Gene-disease association. Based on the PubMed database, the frequency with which CDCA7 was reported to be associated with disease was collected, and the histogram of the top 20 diseases associated with CDCA7 is shown in Fig. 2. Notably, CDCA7 was most commonly reported in neoplasms, but its association with CRC has not yet been reported. Hence, there is still more research that is needed to investigate the expression and significance of CDCA7 in CRC.

Regulatory relationships. As shown in Table III, there were numerous regulatory factors associated with CDCA7, such as hsa-let-7b-5p and human leukocyte antigen (HLA) complex P5 (HCP5), indicating that this gene may act as a transcriptional regulator.

Transcription factor prediction. The transcription factors bound to the promoter region of CDCA7 were predicted based on their positional binding relationship. It was identified that upstream-binding protein 1 (LBP1), zinc finger 5 (ZF5) and interferon regulatory factor 1 (IRF1) were closely associated with CDCA7 (Fig. 3).

Gene expression in tumors. The expression levels of CDCA7 in each sample were analyzed by the RNA-SEQv2 data derived from TCGA database. The results showed that CDCA7 expression was mainly upregulated in tumor tissues compared to adjacent normal tissues (Fig. 4).

High expression of CDCA7 in CRC tissues. Immunohistochemistry SP staining revealed that the expression levels of CDCA7 were higher in CRC tissues $(75.96 \%)$ compared with in adjacent normal colorectal tissues $(26.92 \% ; \mathrm{P}<0.0001$; Fig. 5A). In addition, the RT-qPCR results indicated that the expression levels of CDCA7 were significantly upregulated among $15 \mathrm{CRC}$ tissues compared to adjacent normal colorectal tissues $(\mathrm{P}=0.044$; Fig. 5B). Furthermore, western blotting revealed that similar increased levels of CDCA7 protein were observed in $15 \mathrm{CRC}$ tissues $(\mathrm{P}=0.016$; Fig. 5C and D).

High expression of $C D C A 7$ in $C R C$ cell lines. CDCA7 expression levels were relatively higher in $\mathrm{CRC}$ cell lines compared to NCM460 (a normal colonic epithelial cell line), as revealed by RT-qPCR (Fig. 6A) and western blotting (Fig. 6B and C) analyses, suggesting that CDCA7 serves a role in CRC.

CDCA7 expression is associated with the clinical progression of $C R C$. The results of RT-qPCR and western blotting indicated that CDCA7 expression levels were significantly increased in human CRC. To further examine the potential association 

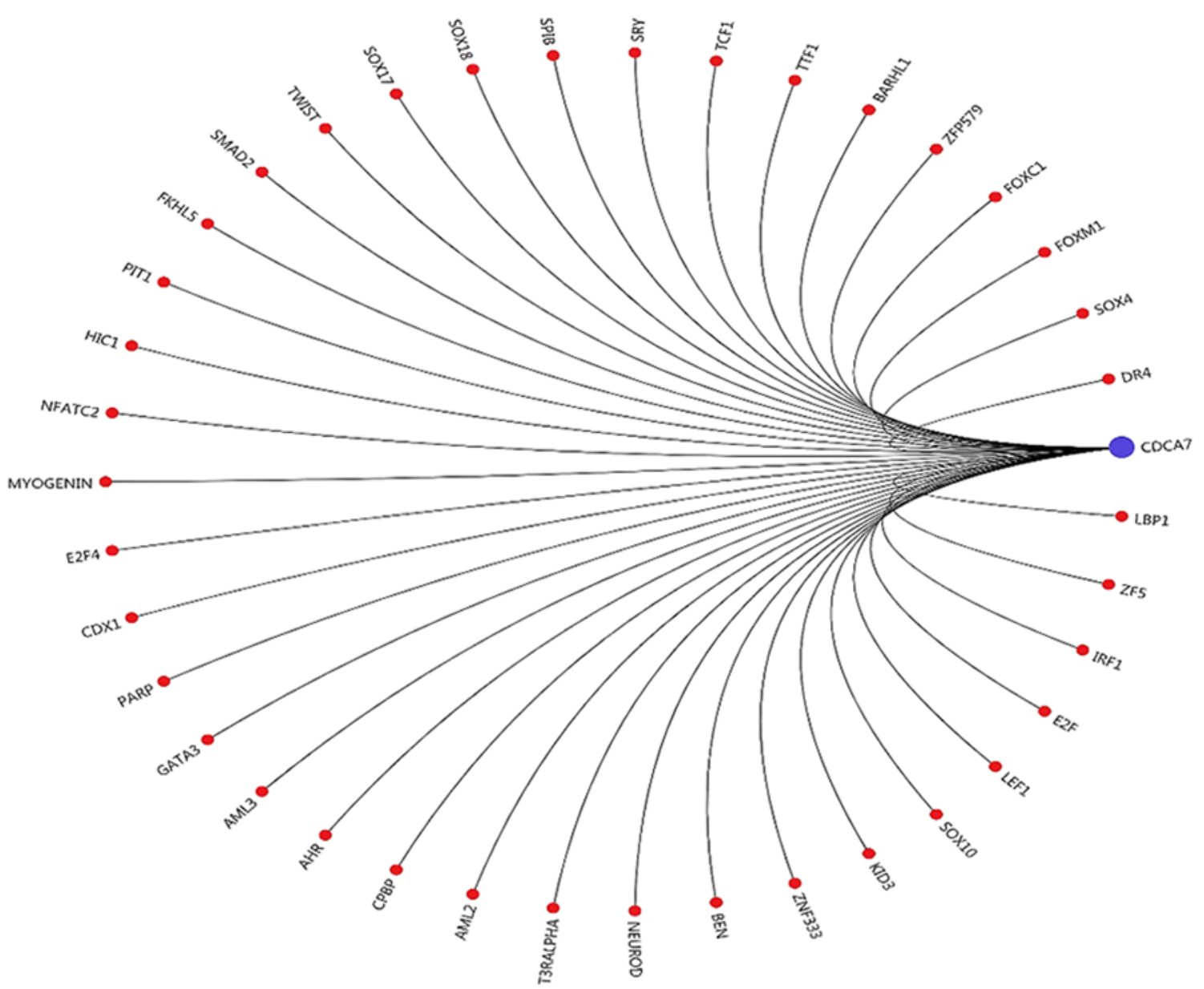

Figure 3. Transcription factors of CDCA7. Red color represents high recommendation. CDCA7, cell division cycle associated protein 7.

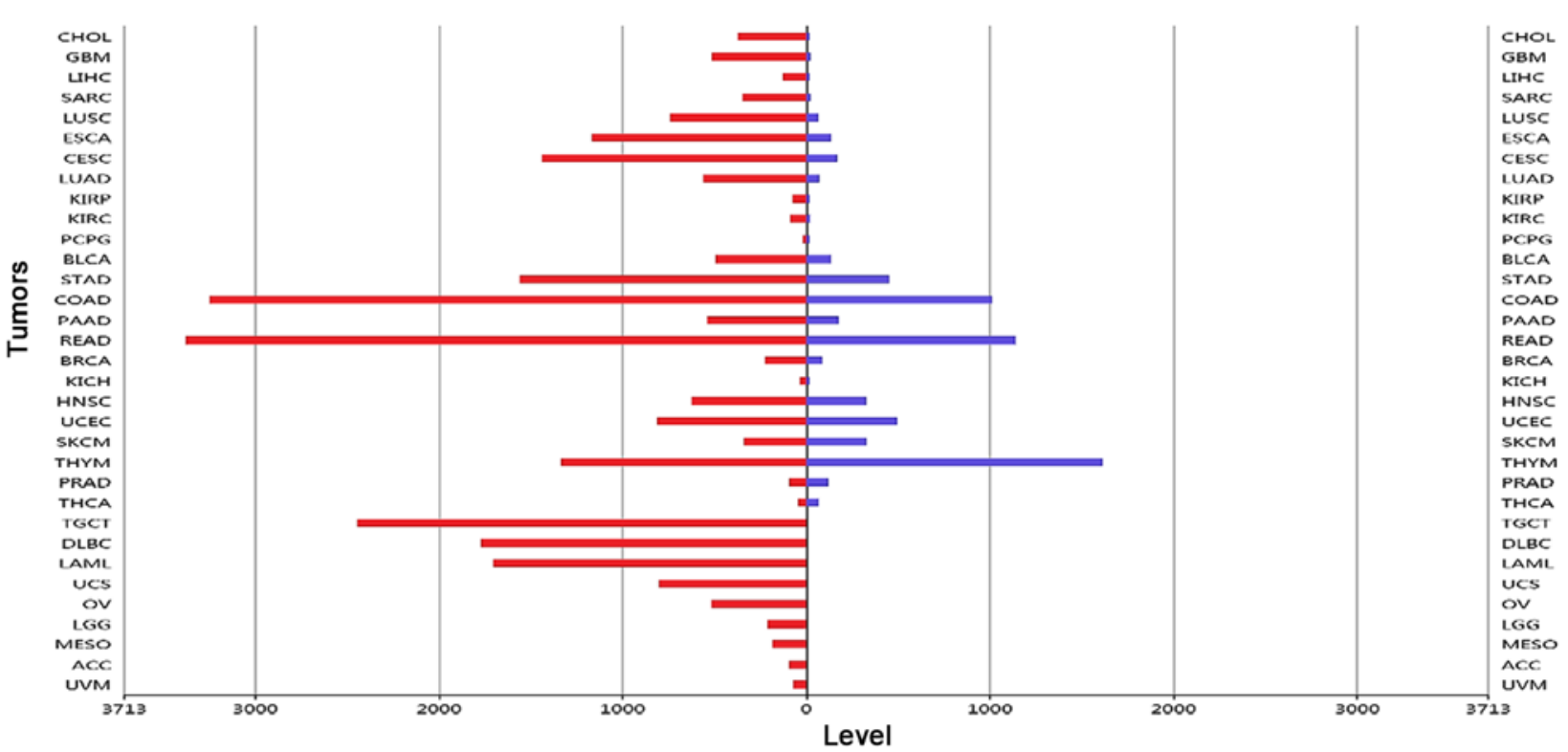

Figure 4. Expression levels of cell division cycle associated protein 7 in various types of cancer. Red represents tumor tissues, while blue represents normal tissues.

between CDCA7 expression and CRC clinicopathological features, the expression levels of CDCA7 were detected in 104 CRC tissues and their paired adjacent non-tumor tissues via immunohistochemistry staining. The positive expression rates of CDCA7 were $26.92 \%(28 / 104)$ and $75.96 \%(79 / 104)$ in normal and CRC tissues, respectively, with a statistically significant difference $(\mathrm{P}<0.0001)$ (data not shown). CDCA7 expression levels in 104 pairs of CRC tissues were catego- 

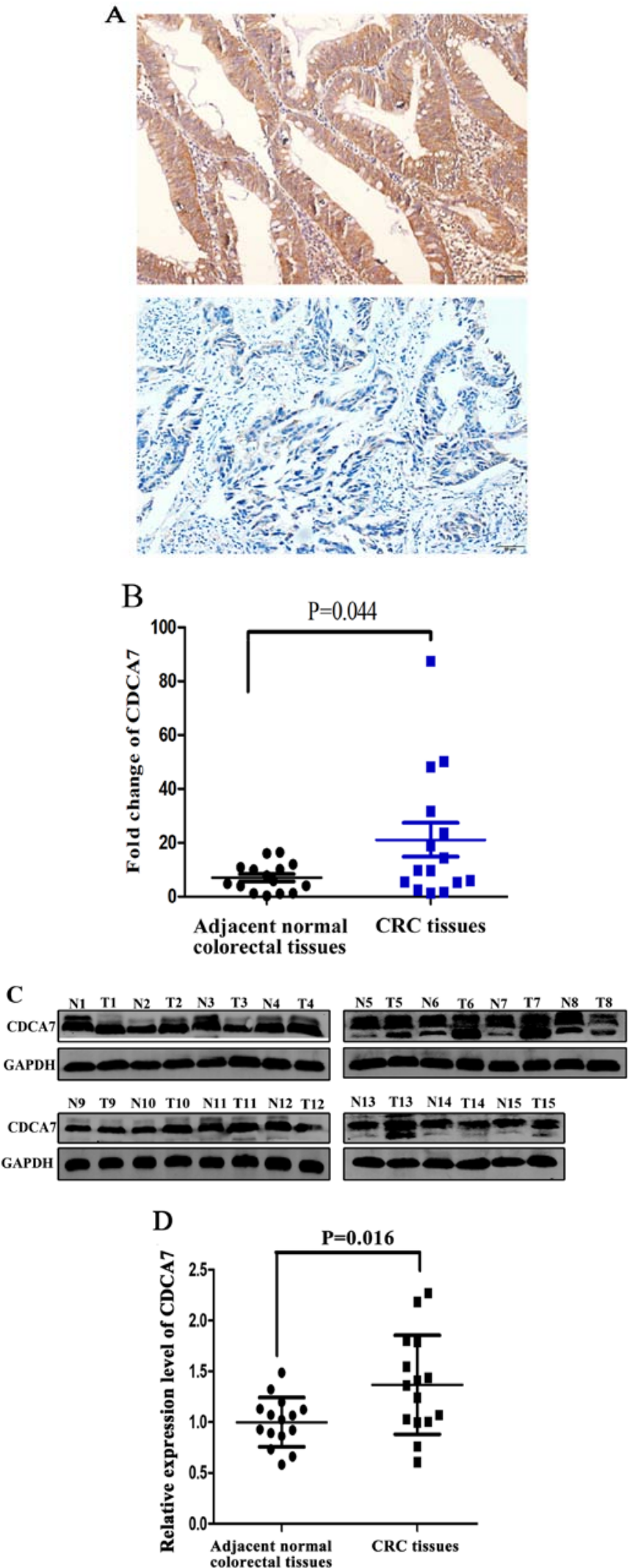

Figure 5. Levels of CDCA7 in CRC tissues. (A) Immunohistochemistry streptavidin peroxidase staining was conducted to determine the levels of CDCA7 in CRC tissues and paired adjacent normal colorectal tissues (magnification, x400). (B) Relative mRNA expression levels of CDCA7 in 15 pairs of $\mathrm{CRC}$ tissues and adjacent normal colorectal tissues were assessed by reverse transcription-quantitative PCR and (C) western blotting. (D) Relative protein levels of CDCA7 in $15 \mathrm{CRC}$ tissues and their paired adjacent normal colorectal tissues. GAPDH was used as the internal control. Data were examined by t-test and are presented as the mean \pm SD. CDCA7, cell division cycle associated protein 7; CRC, colorectal cancer
Table IV. Association between CDCA7 expression and clinicopathological characteristics of patients with CRC.

\begin{tabular}{|c|c|c|c|c|}
\hline \multirow{2}{*}{$\begin{array}{l}\text { Clinicopathological } \\
\text { characteristics }\end{array}$} & \multirow{2}{*}{$\begin{array}{l}\text { Number of } \\
\text { cases }\end{array}$} & \multicolumn{2}{|c|}{ CDCA7 } & \multirow[b]{2}{*}{ P-value } \\
\hline & & Low & High & \\
\hline Sex & & & & 0.533 \\
\hline Male & 61 & 16 & 45 & \\
\hline Female & 43 & 9 & 34 & \\
\hline Age (years) & & & & 0.369 \\
\hline$<60$ & 58 & 12 & 46 & \\
\hline$\geq 60$ & 46 & 13 & 33 & \\
\hline Tumor size & & & & 0.217 \\
\hline$<5 \mathrm{~cm}$ & 64 & 18 & 46 & \\
\hline$\geq 5 \mathrm{~cm}$ & 40 & 7 & 33 & \\
\hline Invasion depth & & & & 0.002 \\
\hline Submucosal & 17 & 9 & 8 & \\
\hline Below the muscle layer & 87 & 16 & 71 & \\
\hline Differentiation & & & & 0.318 \\
\hline High & 12 & 3 & 9 & \\
\hline Moderate & 78 & 21 & 57 & \\
\hline Poor & 14 & 1 & 13 & \\
\hline TNM stage & & & & \\
\hline I-II & 54 & 20 & 34 & 0.001 \\
\hline III-IV & 50 & 5 & 45 & \\
\hline Lymph node metastasis & & & & 0.038 \\
\hline Negative & 65 & 20 & 45 & \\
\hline Positive & 39 & 5 & 34 & \\
\hline Distant metastasis & & & & 0.019 \\
\hline Negative & 89 & 25 & 64 & \\
\hline Positive & 15 & 0 & 15 & \\
\hline
\end{tabular}

CDCA7, cell division cycle associated protein 7; TNM stage, tumor-node-metastasis.

rized into high-expression $(n=79)$ and low-expression $(n=25)$ groups. As summarized in Table IV, high expression levels of CDCA7 were significantly associated with CRC invasion depth $(\mathrm{P}=0.002)$, lymph node metastasis $(\mathrm{P}=0.038)$, tumor-node-metastasis stage $(\mathrm{P}=0.001)$ and distant metastasis $(\mathrm{P}=0.019)$. However, no significant differences were found in sex $(\mathrm{P}=0.533)$, age $(\mathrm{P}=0.369)$, tumor size $(\mathrm{P}=0.217)$ and CRC differentiation $(\mathrm{P}=0.318)$ between high and low CDCA7 expression groups. Collectively, these results indicated that CDCA7 overexpression may be involved in the progression of CRC.

High expression of CDCA7 predicts poor prognosis in patients with CRC. The association between CDCA7 expression and overall survival in patients with CRC was evaluated by Kaplan-Meier analysis. As shown in Fig. 7, high CDCA7 expression group was significantly associated with shorter survival times as compared with the low CDCA7 expression group $(\mathrm{P}=0.012)$. 

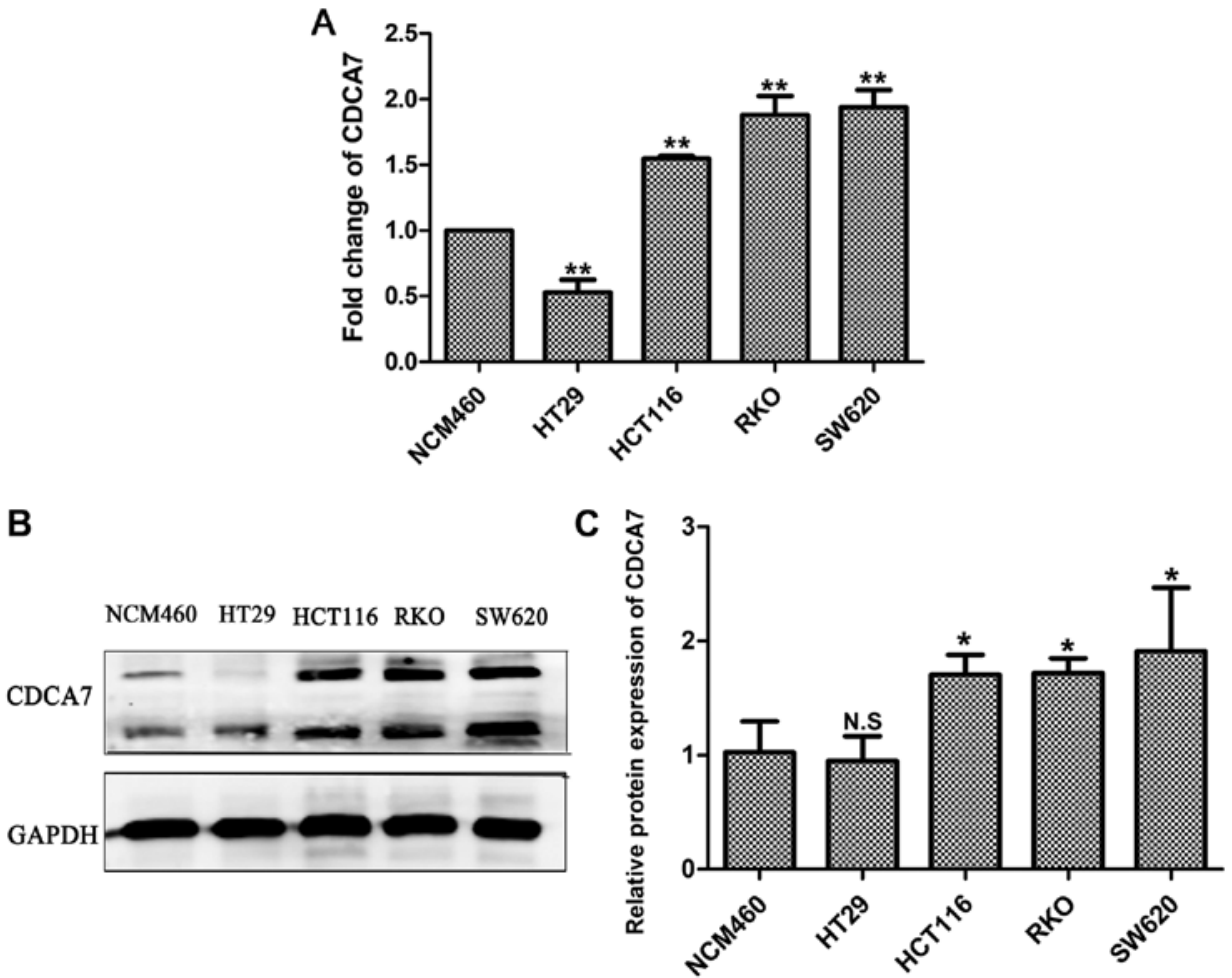

Figure 6. Expression levels of CDCA7 mRNA and protein in colorectal cancer cell lines. (A) mRNA expression levels of CDCA7 were detected by fluorescence-based quantitative PCR. (B) Western blotting showing the protein expression levels of CDCA7; (C) corresponding histogram. GAPDH was used as the internal control; expression value of CDCA7 was set to 1 in the NCM460 group. The data were analyzed by the one-way ANOVA and Bonferroni's post hoc test and are presented as the mean $\pm \mathrm{SD} .{ }^{*} \mathrm{P}<0.05,{ }^{* *} \mathrm{P}<0.01$ vs. NCM460 group. CDCA7, cell division cycle associated protein 7; N.S., non-significant.

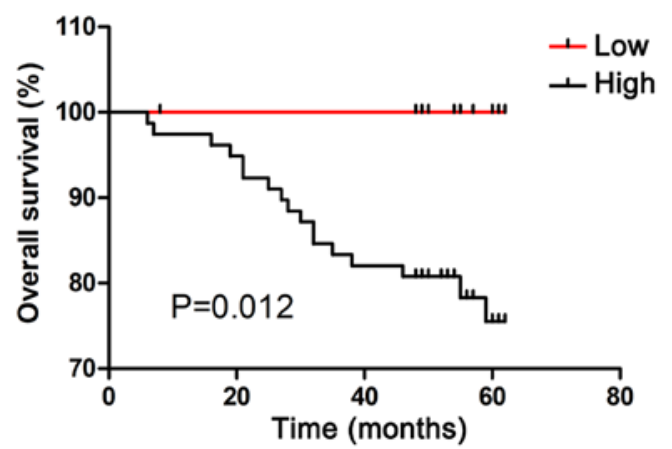

Figure 7. CDCA7 overexpression predicts poor prognosis in patients with colorectal cancer. Kaplan-Meier survival curves were established for both high and low CDCA7 expression groups. High CDCA7 expression group $(\mathrm{n}=79)$ exhibited a poorer prognosis $(\mathrm{P}=0.012)$ compared to the low CDCA7 expression group $(\mathrm{n}=25)$. CDCA7, cell division cycle associated protein 7.

\section{Discussion}

$\mathrm{CRC}$ is the most common form of gastrointestinal cancer, and one of the leading causes of cancer-related mortality globally $(21,22)$. Although clinicopathological parameters have been used to predict the clinical outcome of CRC in patients, these classification indicators are not precise enough to predict the prognosis of patients with CRC. Therefore, it is essential to identify novel tumor biomarkers and therapeutic targets for early diagnosis and treatment of CRC. This study focused on determining the expression levels and clinical role of CDCA7 in CRC.
CDCA7 is a family member of the cell division cycle proteins, which are mainly expressed in the cell nucleus (9). In the present study, the results of immunohistochemical staining demonstrated that CDCA7 was expressed primarily in the nucleus, and at least partly in cytoplasm. A previous study has demonstrated that CDCA7 is a Myc target gene, which participates in Myc-mediated tumor transformation and eventually leads to tumor occurrence (9). Mitotic stimulation can induce the activation of Myc to promote cell cycle progression $(23,24)$. Moreover, CDCA7 is positively expressed in solid tumors, along with high Myc levels (17). These findings indicated that CDCA7 may promote tumor growth progression with Myc. In a previous study, CDCA7 was identified as a novel transcription factor E2F transcription factor 1 (E2F1)-induced protein, suggesting that the expression of CDCA7 is activated by both E2F1 and Myc (25). Additionally, other studies have shown that Myc and E2F1 can bind to the CDCA7 promoter in order to upregulate the expression levels of CDCA7 $(9,25)$. These findings suggest that CDCA7 may participate in tumor occurrence via the activation of Myc and E2F1. However, this statement needs further experimental verification.

It is worth noting that disordered cell cycle control is a basic feature of tumor pathogenesis, and there is a large imbalance in cyclic protein regulation in tumor cells (26-28). High CDCA7 expression has been detected in different types of human cancer, indicating that CDCA7 may function as an oncogene. For example, Jiménez-P et al (14) demonstrated that CDCA7 protein is upregulated in Burkitt lymphoma cell lines and tumor tissues, and CDCA7 mRNA levels are 
significantly elevated in numerous $\mathrm{T}$ and $\mathrm{B}$ lymphoma cell lines. In addition, a previous study reported that CDCA7 is overexpressed in the YDOV-151 human ovarian cancer cell line ( $>7$-fold expression) compared with in human ovarian surface epithelial cells (10). Moreover, CDCA7 has been reported to be involved in the occurrence of retinoblastoma, which can be utilized as a biomarker for early diagnosis and treatment of the disease (15). Cheng et al (18) demonstrated that the expression level of CDCA7 is higher in esophageal squamous cell carcinoma compared to normal esophageal tissue. Furthermore, Osthus et al (17) reported that CDCA7 is overexpressed in patients with acute myeloid leukemia, leading to an increased risk of lymphoid malignancies in these patients. Overall, CDCA7 is upregulated in a wide variety of human tumors, and is likely to be associated with cancer progression.

Additionally, previous evidence has suggested that CDCA7 is involved in the proliferation and apoptosis of tumor cells (29). Recently, it has been shown that the lncRNA FGD5-AS1 can promote the proliferation, migration and invasion of CRC cells by upregulating CDCA7 via sponging miR-302e (30). This study also found that FGD5-AS1 can competitively bind with miR-302e to modulate CDCA7, resulting in the induction of CRC cell apoptosis (30). This indicated that $\mathrm{CDCA} 7$ exhibits a transcriptional regulatory function and by being modulated by its upstream target IncRNA, it can affect the progression of CRC. In the present study, GO term analysis revealed that CDCA7 was related to cell proliferation and apoptosis. While a recent report has focused on the molecular mechanisms of CDCA7 and CRC (30), the exact relationship between CDCA7 and CRC still remains largely unknown. Further research into this relationship is needed in the future.

The results of the present study indicated that CDCA7 expression was upregulated in human CRC tissues compared with in adjacent normal tissues. In addition, it was shown that high CDCA7 expression could contribute to advanced tumor progression in patients with CRC. The results of Kaplan-Meier analysis demonstrated that different expression levels of CDCA7 exhibited significant effects on the prognosis of patients with $\mathrm{CRC}(\mathrm{P}=0.012)$. Hence, $\mathrm{CDCA} 7$ could be a reliable marker for predicting tumor progression and survival prognosis in patients with CRC, but further studies are needed in order to validate this. However, this study is limited by the lack of clarification on the specific mechanisms underlying the positive association between CDCA7 and CRC progression. Therefore, further research and clinical trials into CDCA7 are needed in the future.

In conclusion, the results of the present study provided evidence that CDCA7 may be highly expressed in CRC tissues and may be associated with advanced tumor progression. Notably, to the best of our knowledge, this is the first study to investigate the expression of CDCA7 in CRC tissues and cell lines, as well as its relationship with the clinical parameters of tumor progression. In addition, these results demonstrated that high CDCA7 expression contributes to poor prognosis in patients with CRC. Taken together, these results suggested that CDCA7 may serve as a novel biomarker for CRC diagnosis and a reference indicator for the prognosis of patients with CRC.

\section{Acknowledgements}

The authors would like to thank Dr S.Q. Liu, Dr Y.J. Su, Mr. T,Y. Zhang and Mr. Y. Zhu at the Department of Gastroenterology, The Second Affiliated Hospital of Guangxi Medical University for their help and excellent technical support.

\section{Funding}

The current study was funded by the National Nature Science Foundation of China (grant no. 81760516) and the Program for Improvement Scientific Research Ability of Young and Middle-Aged Teachers of Higher Education of Guangxi (grant no. 2017KY0093).

\section{Availability of data and materials}

The datasets used and/or analyzed during the current study are available from the corresponding author on reasonable request.

\section{Authors' contributions}

CL designed the study and revised the manuscript. MQ and JZ contributed to sample collection and performed the bioinformatics analysis. SL and JH performed the experiments, data analysis and wrote the manuscript. All authors read and approved the final manuscript.

\section{Ethics approval and consent to participate}

Informed consent was obtained from patients. The present study was approved by the Protection of Human Ethics Committee of the First Affiliated Hospital of Guangxi Medical University.

\section{Patient consent for publication}

Not applicable.

\section{Competing interests}

The authors declare that they have no competing interests.

\section{References}

1. Torre LA, Freddie B, Siegel RL, Ferlay J, Lortet-Tieulent J and Jemal A: Global cancer statistics, 2012. CA Cancer J Clin 65: 87-108, 2015.

2. Siegel RL, Miller KD and Jemal A: Cancer statistics, 2015. CA Cancer J Clin 65: 5-29, 2015.

3. Ferlay J, Soerjomataram I, Dikshit R, Eser S, Mathers C, Rebelo M, Parkin DM, Forman D and Bray F: Cancer incidence and mortality worldwide: Sources, methods and major patterns in GLOBOCAN 2012. Int J Cancer 136: E359-E386, 2015.

4. Chiu HM, Hsu WF, Chang LC and Wu MH: Colorectal cancer screening in Asia. Curr Gastroenterol Rep 19: 47, 2017.

5. Calon A, Espinet E, Palomo-Ponce S, Tauriello DV, Iglesias M, Céspedes MV, Sevillano M, Nadal C, Jung P, Zhang XH, et al: Dependency of colorectal cancer on a TGF- $\beta$-driven program in stromal cells for metastasis initiation. Cancer Cell 22: 571-584, 2012.

6. Cui G, Cai F, Ding Z and Gao L: MMP14 predicts a poor prognosis in patients with colorectal cancer. Hum Pathol 83: 36-42, 2019. 
7. Ogata H, Goto S, Sato K, Fujibuchi W, Bono H and Kanehisa M KEGG: Kyoto Encyclopedia of Genes and Genomes. Nucleic Acids Res 27: 29-34, 1999.

8. Harris MA, Clark J, Ireland A, Lomax J, Ashburner M, Foulger R, Eilbeck K, Lewis S, Marshall B, Mungall C, et al: The Gene Ontology (GO) database and informatics resource. Nucleic Acids Res 32 (Database Issue): D258-D261, 2004.

9. Prescott JE, Osthus RC, Lee LA, Lewis BC, Shim H, Barrett JF, Guo Q, Hawkins AL, Griffin CA and Dang CV: A novel c-Myc-responsive gene, JPO1, participates in neoplastic transformation. J Biol Chem 276: 48276-48284, 2001.

10. Cho H, Lim BJ, Kang ES, Choi JS and Kim JH: Molecular characterization of a new ovarian cancer cell line, YDOV-151, established from mucinous cystadenocarcinoma. Tohoku J Exp Med 218: 129-139, 2009.

11. Lewis BC, Shim H, Li Q, Wu CS, Lee LA, Maity A and Dang CV: Identification of putative c-Myc-responsive genes: Characterization of rcl, a novel growth-related gene. Mol Cell Biol 17: 4967-4978, 1997.

12. Basu Baul TS, Longkumer I, Duthie A, Singh P, Koch B and Guedes da Silva MFC: Triphenylstannyl ((arylimino)methyl) benzoates with selective potency that induce $\mathrm{G} 1$ and $\mathrm{G} 2 / \mathrm{M}$ cell cycle arrest and trigger apoptosis via ROS in human cervical cancer cells. Dalton Trans 47: 1993-2008, 2018.

13. Wang YC, Chang KC, Lin BW, Lee JC, Lai CH, Lin LJ, Yen Y, Lin CS, Yang SJ, Lin PC, et al: The EGF/hnRNP Q1 axis is involved in tumorigenesis via the regulation of cell cycle-related genes. Exp Mol Med 50: 1-14: 2018

14. Jiménez-P R, Martín-Cortázar C, Kourani O, Chiodo Y, Cordoba R, Domínguez-Franjo MP, Redondo JM, Iglesias T and Campanero MR: CDCA7 is a critical mediator of lymphomagenesis that selectively regulates anchorage-independent growth. Haematologica 103: 1669-1678, 2018.

15. Wang QL, Chen X, Zhang MH, Shen QH and Qin ZM: Identification of hub genes and pathways associated with retinoblastoma based on co-expression network analysis. Genet Mol Res 14: 16151-16161, 2015.

16. Albulescu R: Elevated cyclin B2 expression in invasive breast carcinoma is associated with unfavorable clinical outcome. BMC Cancer 3: 1, 2013

17. Osthus RC, Karim B, Prescott JE, Smith BD, McDevitt M, Huso DL and Dang CV: The Myc target gene JPO1/CDCA7 is frequently overexpressed in human tumors and has limited transforming activity in vivo. Cancer Res 65: 5620-5627, 2005.

18. Cheng C, Zhou Y, Li H, Xiong T, Li S, Bi Y, Kong P, Wang F, $\mathrm{Cui} \mathrm{H}, \mathrm{Li} \mathrm{Y}$, et al: Whole-genome sequencing reveals diverse models of structural variations in esophageal squamous cell carcinoma. Am J Hum Genet 98: 256-274, 2016.

19. Tate JG, Bamford S, Jubb HC, Sondka Z, Beare DM, Bindal N, Boutselakis H, Cole CG, Creatore C, Dawson E, et al: COSMIC: The catalogue of somatic mutations in cancer. Nucleic Acids Res 47: D941-D947, 2019.
20. Livak KJ and Schmittgen TD: Analysis of relative gene expression data using real-time quantitative PCR and the 2(-Delta Delta C(T)) method. Methods 25: 402-408, 2001

21. Bray F, Ferlay J, Soerjomataram I, Siegal RL, Torre LA and Jemal A: Global Cancer Statistics 2018: GLOBOCAN Estimates of Incidence and Mortality Worldwide for 36 Cancers in 185 Countries, CA Cancer J Clin 68 (6), 394-424, 2018.

22. GBD 2015 Mortality and Causes of Death Collaborators. Global, regional, and national life expectancy, all-cause mortality, and cause-specific mortality for 249 causes of death, 1980-2015: a systematic analysis for the Global Burden of Disease Study 2015. Lancet 388 (10053), 1459-1544, 2016.

23. Conover CA and Bale LK: Insulin-like growth factor I induction of c-myc expression in bovine fibroblasts can be blocked by antecedent insulin receptor activation. Exp Cell Res 238: 122-127, 1998.

24. Kitaura H, Shinshi M, Uchikoshi Y, Ono T, Iguchi-Ariga SM and Ariga $\mathrm{H}$ : Reciprocal regulation via protein-protein interaction between c-Myc and p21(cip1/waf1/sdi1) in DNA replication and transcription. J Biol Chem 275: 10477-10483, 2000.

25. Goto Y, Hayashi R, Muramatsu T, Ogawa H, Eguchi I, Oshida Y, Ohtani K and Yoshida K: JPO1/CDCA7, a novel transcription factor E2F1-induced protein, possesses intrinsic transcriptional regulator activity. Biochim Biophys Acta 1759: 60-68, 2006.

26. Zhang J, Sun Z, Han Y, Yao R, Yue L, Xu Y and Zhang J: Rnf2 knockdown reduces cell viability and promotes cell cycle arrest in gastric cancer cells. Oncol Lett 13: 3817-3822, 2017.

27. Lee J, Choi BY and Keum YS: Acetonitrile extract of Salvia miltiorrhizaRadix exhibits growth-inhibitory effects on prostate cancer cells through the induction of cell cycle arrest and apoptosis. Oncol Lett 13: 2921-2928, 2017.

28. Lu J, Chen X, Qu S, Yao B, Xu Y, Wu J, Jin Y and Ma C: Oridonin induces $\mathrm{G}_{2} / \mathrm{M}$ cell cycle arrest and apoptosis via the PI3K/Akt signaling pathway in hormone-independent prostate cancer cells. Oncol Lett 13: 2838-2846, 2017.

29. Gill RM, Gabor TV, Couzens AL and Scheid MP: The MYC-associated protein CDCA7 is phosphorylated by AKT to regulate MYC-dependent apoptosis and transformation. Mol Cell Biol 33: 498-513, 2013.

30. Li D, Jiang X, Zhang X, Cao G, Wang D and Chen Z: Long noncoding RNA FGD5-AS1 promotes colorectal cancer cell proliferation, migration, and invasion through upregulating CDCA7 via sponging miR-302e. In Vitro Cell Dev Biol Anim 55: $577-585,2019$.

(i) $(-)$ This work is licensed under a Creative Commons Attribution-NonCommercial-NoDerivatives 4.0 International (CC BY-NC-ND 4.0) License. 